\title{
Ultrasound Versus the Landmark Technique: A Prospective Randomized Comparative Study of Internal Jugular Vein Cannulation in an Intensive Care Unit
}

\author{
Shrestha BR, Gautam B \\ Department of Anesthesiology and ICU, Kathmandu Medical College-Teaching Hospital, Kathmandu, Nepal
}

\section{ABSTRACT}

Objective: The aim of this study is to find out if an ultrasound technique has advantages over the conventional landmark technique.

Methods: This is a prospective randomized comparative study on 120 patients requiring central venous cannulation of the right internal jugular vein. The study comprised of two groups: ultrasound and landmark groups, each consisting of 60 patients. The outcome measures were compared between the groups.

Results: Cannulation of the internal jugular vein was successful in 58 patients in the ultrasound group and in 53 in the landmark group. The number of attempts was $1.5(1-3)$ and $2(1-3)$ in the ultrasound and landmark group respectively $(\mathrm{p}=0.001)$. The time taken for the successful cannulation was $4.9 \pm 1.7$ minutes in the ultrasound approach and $8.0 \pm 2.8$ minutes in the landmark approach $(\mathrm{p}=0.00)$. The internal jugular vein diameter in the supine position was $11.2 \pm 1.5 \mathrm{~mm}$ which increased to $15.04 \pm 1.5 \mathrm{~mm}$ with a 15 degrees head-down position in the USG group $(\mathrm{p}=$ 0.001). The first attempt success rate was 39/60 (63\%) in the ultrasound group and 19/60 (32\%) with the landmark technique. The seven (12\%) failure cases in the landmark group were rescued by the ultrasound technique. Inadvertent carotid artery puncture occurred in 2/60 (3\%) and 6/60 (10\%) of patients in the ultrasound and land mark group respectively.

Conclusions: Ultrasound improves success rate, minimizes cannulation time and complications during internal jugular vein cannulation. It can be employed as a rescue technique in cases of a failed landmark technique.

Keywords: cannulation, central, landmark, technique, ultrasound

\section{INTRODUCTION}

Placement of a central venous catheter remains a common procedure during the management of patients in the intensive and critical care units. The external landmark method is still the technique of choice in many critical care units. Central vascular access in patients admitted to intensive care units (ICU) is required for fluid therapy, total parenteral nutrition, administration of cardiotonic vasoactive drugs and chemotherapy or for continuous haemodynamic monitoring, temporary cardiac pacing, hemodialysis or in cases when peripheral veins are not easily accessible.

The landmark technique is still the method of choice for central venous cannulation (CVC) in our context due to reasons including an unavailability of equipments and a lack of training facility. With the evolution of newer technology, the blind technique is gradually being replaced by under-vision ultrasound assisted CVC.

\footnotetext{
correspondence:

Dr.Babu Raja Shrestha

Department of Anesthesiology and ICU

KMC-TH, Sinamangal, Kathmandu, Nepal

E-mail: barashrestha@yahoo.co.in
} 
A method for percutaneous cannulation of the internal jugular vein (IJV) using external landmarks was first described in 1966. Even though the benefits of ultrasound (USG) guidance for locating the central veins was recognized as far back as $1978^{1}$, the first report of ultrasound-guided CVC came into existence in 1987. Since then, abundant clinical reports emerged in the literature showing the efficacy and safety of this procedure over the traditional one. Recognizing this fact, the National Institute of Clinical Excellence in the United Kingdom has recommended the use of USG guidance for CVC as one of the mandatory practices to improve patient care. ${ }^{2}$ Ultrasound has become an integral tool for high precision CVC, providing a rapid procedure as well as less complication in the ICUs.

The main purpose of this study was to compare the landmark and USG techniques for CVC with regards to success rate, incidence of complications, time taken to accomplish the procedure and possibility of USG assistance in cases of failed multiple attempts with the conventional landmark method.

\section{METHOD}

\section{Patient selection}

This was a prospective, randomized comparative study performed in the ICU at the Kathmandu Medical College-Teaching Hospital, Nepal. Consecutive patients from February 2010 to October 2010 who required CVC during their ICU stay were enrolled in the study. Informed consent for the procedure was taken from the patients and/or relatives. Clearance from the institutional ethical committee was obtained for the study. Patients were randomized into either of the groups using a lottery method. The exclusion criteria included clinical evidence of coagulopathy, prolonged prothrombin time and INR, platelets count less than $75000 / \mathrm{cmm}$, history of anticoagulant therapy, documentaed inherited coagulation disorder and dissiminated intravascular coagulation, age below 17 years and local infection at the site of cannulation.

The first group of patients underwent USG-guided CVC in the right IJV; and in the second group, the right IJV was cannulated employing the landmark technique.

\section{Intervention}

The performer of either technique in this study was the same person. In the USG group, a portable ultrasound scanner with superficial linear probe of $6.5 \mathrm{~cm}$ length and frequency of $8.0 \mathrm{MHz}$ (Toshiba, PLG-805S) was used. Under all aseptic precautions, the puncture site was infiltrated with $1 \%$ lignocaine. On linear probe of the ultrasound, ultrasonic gel was applied which was then covered with a sterile transparent plastic sheath and fixed with sterile rubber bands. The patient was positioned in the supine and the head turned to the other side.

The transducer was placed perpendicular to the vessels at the apex of the triangle formed by the two heads of sternocleidomastoid muscle and clavicle. The IJV was identified as an oval thin walled hypoechoic compressible structure lying lateral and superficial to non-compressible pulsating carotid artery. The position of the IJV in relation to the carotid artery was recorded. The IJV picture was then centered in the acoustic window. At the cricoid level, the diameter of IJV was recorded in the supine and Trendelenburg position (15 degrees) in the USG group. The number of patients who were ventilated mechanically was recorded in each group. In case of mechanically ventilated patients, the positive end expiratory pressure level if previously added was zeroed before obtaining the IJV diameters. An introducer needle with an attached syringe was inserted under the probe at an angle of 45 degrees using the out-of-plane method. ${ }^{3}$ The movement of the needle tip and the change of the shape of the vein was carefully observed. The tip of the needle lying intravascularly was visualized clearly on the image and the free flow of blood upon aspiration confirmed the correct position of the needle. The guide-wire was then passed and the catheter rail-roaded over it after dilatation of the tissue plane.

The time to successful completion of the cannulation in the study was the time from the skin puncture to blood aspiration via the catheter immediately following the guide-wire removal. An attempt was considered unsuccessful if complete withdrawal of the puncturing needle out of skin surface once it was pricked was required. The procedure was regarded as a failure if the operator was unable to cannulate the vein in three attempts.

In the landmark group, IJV cannulation was performed using the standard guidelines for the central method. In case of an unsuccessful cannulation after three attempts, it was rescued by ultrasound-guided cannulation.

Post-procedural chest X-ray was ordered to ascertain the position of the distal catheter tip and rule out any possible complications like pneumothorax and haemothorax in both groups. At the end of the procedure, all data including patient demographics, number of attempts, time to cannulation, failure rate, rescued number and complications encountered were recorded independently by another specialist. 
Shrestha et.al.Ultrasound Versus the Landmark Technique in Central Vein Cannulation.

\section{Statistical analysis}

SPSS, 14.0 for windows evaluation version (SPSS Inc. Chicago, Illinois) was used for statistical analysis. The means of continuous variables were compared using the Student's't'-test and categorical variables were compared using ANOVA test. A $p$ value of less than 0.05 was considered statistically significant.

\section{RESULT}

Cannulation of IJV was successful in 58 patients in the USG group and in 53 in the landmark group (97\% vs 88 $\%)$. The number of attempts was $1.5(1-3)$ and 2.0 (1 - 3) in the ultrasound and landmark group respectively $(p=0.001)$. The time taken for successful cannulation was $4.9 \pm 1.7$ minutes in the ultrasound approach and $8.0 \pm 2.8$ minutes in the landmark approach $(p$ $=0.00$ ). The IJV diameter in the supine position was $11.2 \pm 1.5 \mathrm{~mm}$ which increased to $15.04 \pm 1.5 \mathrm{~mm}$ with a 15 degrees head-down position in the USG group $(p=0.001)$. The first attempt success rate was $39 / 60$ (63\%) in the USG group and 19/60 (32\%) with the landmark technique. The seven (12\%) failure cases in the landmark group were later successfully cannulated by the ultrasound technique. Carotid artery puncture occurred in $2 / 60(3 \%)$ and $6 / 60(10 \%)$ of patients in the ultrasound and land mark group, respectively. Carotid artery puncture and hematoma formations were two major complications seen during or after the procedure. No patient in either group of our study had complications like pneumothorax and haemothorax.

Table 2. Outcome measures. The values are mean or number (SD) or (\%).

\begin{tabular}{|c|c|c|c|}
\hline & USG group & Landmark roup & p value \\
\hline $\begin{array}{l}\text { Time taken, min, } \\
\text { mean (range) }\end{array}$ & $5.0(2-12)$ & $8.0(4-15)$ & 0.001 \\
\hline $1^{\text {st }}$ attempt, n (\%) & $38(63 \%)$ & $19(32 \%)$ & \multirow{4}{*}{0.001} \\
\hline $2^{\text {nd }}$ attempt & $17(28 \%)$ & $21(35 \%)$ & \\
\hline $3^{\text {rd }}$ attempt & $3(5 \%)$ & $13(22 \%)$ & \\
\hline No. of attempts (SD) & $1.53 \pm 0.725$ & $2.0 \pm 0.80$ & \\
\hline \multirow{3}{*}{$\begin{array}{l}\text { Overall success } \\
\text { Overall failure, n (\%) }\end{array}$} & $58(97 \%)$ & $53(88 \%)$ & \multirow[b]{2}{*}{-} \\
\hline & 2 (3 \%) & $7(12 \%)$ & \\
\hline & & & \\
\hline \multirow{3}{*}{$\begin{array}{l}\text { IJV diameter, }(\mathrm{mm}) \\
\text { mean } \pm \mathrm{SD} \\
\text { Supine vs. } \\
\text { head down }\end{array}$} & $11.20 \pm 1.46$ & \multirow{3}{*}{-} & \multirow{3}{*}{0.001} \\
\hline & & & \\
\hline & $15.05 \pm 1.49$ & & \\
\hline \multicolumn{3}{|l|}{ Complications, n (\%): } & \multirow{3}{*}{-} \\
\hline Carotid artery puncture & $2(3 \%)$ & $6(10 \%)$ & \\
\hline Hematoma & $1(2 \%)$ & $5(8 \%)$ & \\
\hline Pneumothorax & 0 & 0 & \\
\hline Haemothorax & 0 & 0 & \\
\hline Catheter related infections & 0 & 0 & \\
\hline
\end{tabular}

\section{DISCUSSION}

This study shows the superiority of ultrasound guidance as compared to the landmark technique for cannulation of the right IJV. Traditionally, the site of needle placement is determined by using palpable or visible anatomic structures with the known relationships to the desired vein as landmarks and employing the historically known Seldinger's method of introducing the catheter percutaneously into the vascular channels ${ }^{4}$. Previous studies have established the benefits of ultrasound guidance for CVC in different clinical settings like intensive care units, operative rooms and emergency departments ${ }^{5}$.

The IJV cannulation was successful in the first attempt in $63 \%$ of patients in the USG group as compared 
to $32 \%$ in the landmark group in our study which is comparable to the study performed by Denys BG, et al. ${ }^{6}$ Cannulation of the IJV was successful in $97 \%$ in the USG group and in $88 \%$ of the patients while using the landmark technique in the current study. Our success rate in the USG group was comparable with the findings of Palepu G B et al where overall success rate of $98 \%{ }^{7}$.

Table 1. Patient demographic

\begin{tabular}{lcc}
\hline & USG Group & $\begin{array}{r}\text { Landmark } \\
\text { Group }\end{array}$ \\
\hline Sex (M:F) & $31: 29$ & $27: 33$ \\
Age (yrs), mean \pm SD & $39 \pm 15$ & $38 \pm 15$ \\
$\begin{array}{l}\text { Mechanical ventilation, } \\
\mathrm{n}(\%)\end{array}$ & $26(43 \%)$ & $28(47 \%)$ \\
\hline
\end{tabular}

The time consumed for successful cannulations in various studies are different depending upon the number of cases and the expertise of the performers. Pozzoli $\mathrm{M}$ et $\mathrm{al}^{8}$ found the time to perform cannulation was not significantly different using either approaches. However, Denys BG et $\mathrm{al}^{6}$ had taken the end point of the time spent as from skin to vein puncture, but not till cannulation of the vein, and found the average access time (skin to vein) was $9.8(2-68)$ seconds by the USG approach and $44.5(2-1,000)$ seconds by the landmark approach ( $p<0.001$ ). In the study conducted by Cazzojjo $\mathrm{M}$ et al in 196 patients; 105 patients underwent US-guided CVC and 91 patients had CVC without an US guide. The average time to perform CVC was shorter with the US guide (4 vs 7 min), which was comparable to our results. In terms of success rate also, our result appeared similar to the study by Cazzojjo et al $(98.09 \%$ vs $91.2 \%$, p < $0.025)^{9}$

There were six carotid artery punctures and five hematomas in the landmark group and two carotid artery punctures and one hematoma in the USG group. In the study conducted by Turker $\mathrm{G}$ et al, the overall complication rate was significantly higher in the landmark group than in the USG-guided group. Carotid puncture rate and hematoma were more frequent in the landmark group than in the USG-guided group ( $p<$ $0.05)^{10}$. Another report of a similar kind of complication when the ultrasound was used in paediatric ICU cases is by Froehlich C D et al ${ }^{11}$ with inadvertent artery punctures in the ultrasound group $(8.5 \%$ vs $19.4 \%$, $\mathrm{p}=0.03)$. The rates of major and minor mechanical complications can be as high as $10 \%$ in the landmark technique ${ }^{12}$. The common complications are arterial puncture (10.6 - $13 \%)$ and hematoma formation (4 - $8.4 \%)$ in the conventional landmark technique. ${ }^{13}$ Despite these possible complications USG-guided
CVC cannulation was successful in $100 \%$ of cases (450 patients) whereas 425 cases (94.4\%) were successfully cannulated using the landmark technique in the study conducted in one of the ICUs from Greece showing the superiority of the USG technique over the blind landmark method ${ }^{14}$.

The growing use of ultrasound for CVC is based on clinical evidence of reduced failure rates and minimal complications compared to the landmark techniques ${ }^{15}$.

The mean number of attempts required for successful IJV catheter insertion was $1.53 \pm 0.72$ in the USG group and $2.00 \pm 0.80$ in the landmark group ( $p<$ 0.05 ) in this study which is also consistent with the study performed by Turker et $\mathrm{al}^{10}$. The risk of procedural and post-procedural complications increases with the number of insertion attempts ${ }^{16}$. It is apparent that complications may still occur in the ultrasound technique, but with more reduced frequency than in the landmark technique. This may be related to different factors like skills and learning curves of performers or patient-related factors.

The mean right IJV diameter in the supine position was $11.200 \pm 1.462 \mathrm{~mm}$ which was found to be increased to $15.05 \pm 1.49 \mathrm{~mm}$ in the head-down position in the USG group ( $p=0.001$ ) which compares with the findings of Lamperti et $\mathrm{al}^{17}$. At times, CVC in ICU patients can be extremely difficult; but still, it needs to be accomplished irrespective of patient positions. In patients with clinical conditions that contraindicate the Valsalva maneuver and the head-down position, passively elevating legs may increase the IJV diameter for its easier cannulation ${ }^{18}$

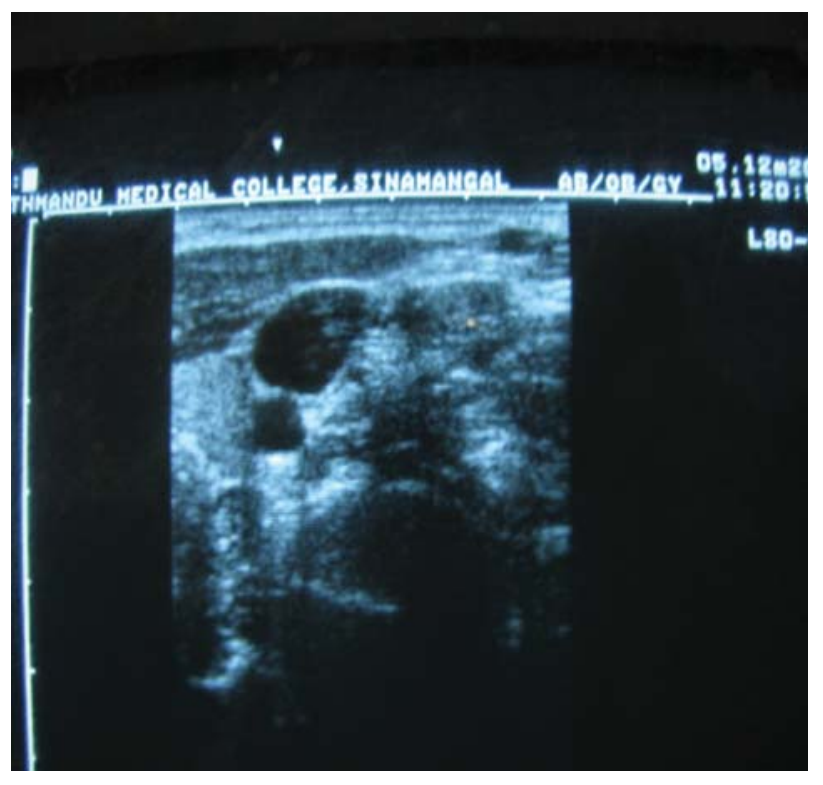

Figure 1. Triangular-shaped IJV in the supine position 


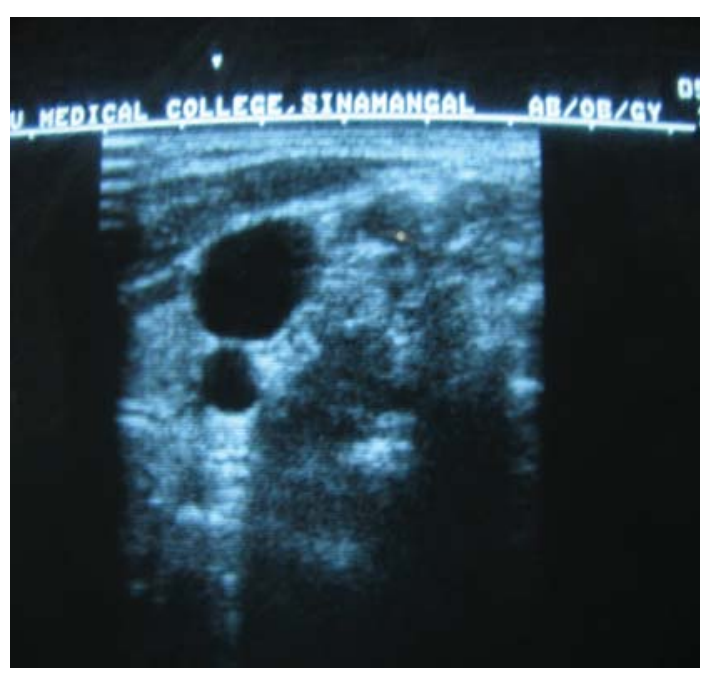

Figure 2. IJV in the supine head-down position with a distended larger-diameter IJV

The position of the IJV with respect to the carotid artery at the level of the cricoid cartilage were found to be lateral (35/60), anterolateal (22/60) and anterior $(5 / 60)$ in the ultrasound group. Such an inter-individual anatomical variation accounts for unsuccessful cannulation of the IJV during the blind techniques. Ultrasound has been found to be a valuable tool to rescue the failure cases. All seven unsuccessful cases in the landmark group were salvaged with the ultrasound assistance. A short neck, an anteriorly-located vein and a collapsed vein due to hypovolemia could have been the main causes of failure rates and a low success rate at the first attempt $(31.56 \%)$ in the landmark group in this study. Ultrasound guidance allows cannulations difficult situations. ${ }^{19}$ Two cases in the USG group could not be cannulated because of the inadvertent carotid artery puncture and subsequent swelling of the neck. The out-of-plane method was preferred over the inplane method for the USG-guided CVC in this study due to the length of the probe itself not being able to be accommodated in the neck region while using the in-plane, long-axis method .

Patient-related factors like Body Mass Index, length and thickness of the neck, and compliance of the patients to the procedure were not included. In addition to these factors, the inability to study the incidence of vascular thromboses is another weakness of the present study.

\section{CONCLUSIONS}

USG-guided CVC of the right IJV increases the firstattempt success rate, decreases the time required for cannulation, reduces the complications, and is a useful alternative to an unsuccessful cannulation using the landmark technique.

\section{REFERENCE}

1. Ullman JI, Stoelting RK. Internal jugular vein location with the Ultrasound Doppler blood flow detector. Anesth Analg. 1978;5:118.

2. National Institute for Clinical Excellence. Guidance on the use of ultrasound locating devices for placing central venous catheters. London: NICE, 2002. NICE Technology Appraisal No 49.

3. Abboud PA, Kendall JI. Ultrasound guidance for vascular access. Emerg Med Clin North Am. 2004; 22:749-73.

4. Greitz T. Sven-Ivar Seldinger. Am J Neuroradiol. 1999;20:1180-1.

5. Hrics P, Willber S, Blanda MP, Callo U. Ultrasound assisted internal jugular vein cannulation in the ED. Am J Emerg Med. 1998;16:401-3.

6. Denys BG, Uretsky BF, Reddy PS. Ultrasound-assisted cannulation of the internal jugular vein: a prospective comparision to the external landmark-guided technique. Circulation. 1993;87:1557-62.

7. Palepu G B, Deven J , Subrahmanyam M, Mohan S. Impact of ultrasonography on central venous catheter insertion in intensive care. Indian J Radiol Imaging. 2009;19(3):191-8.
8. Pozzoli M, Galli F, Capomolla S, Forni G, Cibelli F, Tavazzi L. Usefulness of ultrasonographic techniques in catheterization of the internal jugular vein in patients with chronic heart failure. G Ital Cardiol. 1994;10:1211-21.

9. Cajozzo M ,Quintini G, Cocchiera G, Greco G, Vaglica $R$, Pezzano $G$ et al . Comparision of central venous catheterization with and without ultrasound guide .Transfus Apher Sci. 2004:31:199-202.

10. Turker G, Kaya FN, Gurbet A, Aksu H, Erdogan C, Atlas A. Internal jugular vein cannulation: an ultrasound-guided technique versus landmark-guided technique. Clinics (Sao Paulo). 2009;64:989-92.

11. Froehlich CD, Rigby MR, Rosenberg ES, Li R, Roerig PJ, Easley KA et al. Ultrasound guided central venous catheter placement decreases complications and decreases placement attempts compared with the landmark technique in patients in a pediatric intensive care unit. Crit Care Med. 2009;37:1090-6.

12. Manfred M. Ultrasound guided central venous access is useful for beginners, in children, and when blind cannulation fails. BMJ. 2002;14:1373-4.

13. Martin MJ, Husain FA, Piesman M, Mullenix PS, Steele SR, Andersen $\mathrm{CA}$ et al. Is routine ultrasound guidance for central line placement beneficial? A prospective analysis. Curr Surg. 2004;61:71-4. 
14. Karakitsos D, Labropoulos N, De Groot E, Patrianakos AP, Kaurakli G, Poularas J et al. Real-time ultrasound-guided catheterisation of the internal jugular vein: A prospective comparison with the landmark technique in critical care patients. Crit Care. 2006;10:R162.

15. Randolph AG, Cook DJ, Gonzales CA, Pribble CG. Ultrasound guidance for placement of central venous catheters. A meta-analysis of the literatures. Crit Care Med. 1996;24:2053-8.

16. McGee D, Gould M. Current concepts: Preventing complications of central venous catheterization. $\mathrm{N}$ Engl J Med. 2003;348:1123-33.
17. Lamperti M, Caldiroli D, Cortellazzi P, Vailati D, Pedicelli A, Tosi F et al. Safety and efficacy of ultrasound assistance during internal jugular vein cannulation in neurosurgical infants. Intensive Care Medicine. 2008;34:2100-5.

18. Kim JT, Kim HS, Lim YJ, Bahk JH, Lee KH, Kim CS et al. The influence of passive leg elevation on the cross-sectional area of the internal jugular vein and the subclavian vein in awake adults. Anaesth Intensive Care. 2008;36:65-8.

19. Miller AH, Roth BA, Mills TJ, Woody JR, Longmoor CE, Foster B et al. Ultrasound guidance versus the landmark technique for the placement of central venous catheters in the emergency department. Acad Emerg Med. 2002;9:800-5. 